\title{
ON A CONJECTURE FOR THREE-DIMENSIONAL COMPETITIVE LOTKA-VOLTERRA SYSTEMS WITH A HETEROCLINIC CYCLE
}

\author{
Mats Gyllenberg AND Ping YAN
}

\begin{abstract}
For three-dimensional competitive Lotka-Volterra systems, Zeeman (1993) identified 33 stable equivalence classes. In this paper we show that: in the case of a heteroclinic cycle on the boundary of the carrying simplex of three-dimensional competitive Lotka-Volterra systems (class 27 in Zeeman's classification), the conditions (a) there is a pair of purely imaginary eigenvalues at an interior equilibrium, (b) the first focal value vanishes, $\left(c^{\prime}\right)$ the second focal value vanishes, and (c) the heteroclinic cycle is neutrally stable do not imply (d) the third focal value vanishes. In particular, the conditions (a), (b), $\left(c^{\prime}\right)$, and (c) do not imply that the interior equilibrium is a center. This proves a conjecture by Gyllenberg and Yan (2009).
\end{abstract}

Mathematics subject classification (2000): 34C12, 34C23, 92D25.

Keywords and phrases: competitive Lotka-Volterra system, limit cycles, carrying simplex, heteroclinic cycle.

\section{REFERENCES}

[1] G. Butler, H. I. Freedman and P. Waltman, Uniformly persistent systems, Proc. Amer. Math. Soc., 96 (1986), 425-430.

[2] M. Gyllenberg And P. Yan, On the number of limit cycles for the three-dimensional LotkaVolterra system, Discrete Contin. Dyn. Syst. -B, 11 (2009), 347-352.

[3] M. Gyllenberg And P. YAn, Four limit cycles for a three-dimensional competitive Lotka-Volterra system with a heteroclinic cycle, Computers \& Mathematics with Applications, 58 (2009), 649-669.

[4] M. GYllenberg, P. YAN, AND Y. WANG, A 3D competitive Lotka-Volterra system with three limit cycles: a falsification of a conjecture by Hofbauer and So, Appl. Math. Lett., 19 (2006), 1-7.

[5] M. Gyllenberg, P. YAN, AND Y. WANG, Limit cycles for the competitor-competitor-mutualist Lotka-Volterra systems, Physica D, 221 (2006), 135-145.

[6] M. W. HIRSCH, Systems of differential equations which are competitive or cooperative III: Competing species, Nonlinearity, 1 (1988), 51-71.

[7] M. W. HIRSCH, On existence and uniqueness of the carrying simplex for competitive dynamical systems, Journal of Biological Dynamics, 2:2 (1988), 169-179.

[8] J. Hofbauer And K. Sigmund, Evolutionary Games and Population Dynamics, Cambridge University Press, Cambridge, 1998.

[9] J. Hofbauer And K. Sigmund, Evolutionary game dynamics, Bull. Amer. Math. Soc., 40 (2003), 479-519.

[10] J. HofBAUER AND J. W.-H. So, Multiple limit cycles for three dimensional Lotka-Volterra equations, Appl. Math. Lett., 7 (1994), 65-70.

[11] Z. LU AND Y. Luo, Two limit cycles in three-dimensional Lotka-Volterra systems, Computers \& Mathematics with Applications, 44 (2002), 51-66.

[12] Z. LU AND Y. LuO, Three limit cycles for a three-dimensional Lotka-Volterra competitive system with a heteroclinic cycle, Computers \& Mathematics with Applications, 46 (2003), 231-238.

[13] H. L. Smith, Monotone Dynamical Systems: An Introduction to the Theory of Competitive and Cooperative Systems, Math. Surveys Monogr., Vol. 41, Amer. Math. Soc., Providence (1995).

[14] P. VAN DEN DRIESSCHE AND M. L. ZEEMAN, Three-dimensional competitive Lotka-Volterra systems with no periodic orbits, SIAM J. Appl. Math., 58 (1998), 227-234. 
[15] D. XiaO AND W. LI, Limit cycles for the competitive three dimensional Lotka-Volterra system, J. Differential Equations, 164 (2000), 1-15.

[16] M. L. ZeEman, Hopf bifurcations in competitive three-dimensional Lotka-Volterra systems, Dynamics and Stability of Systems, 8 (1993), 189-217.

[17] Hsiu-Rong Zhu AND H. L. SMith, Stable periodic orbits for a class of three dimensional competitive systems, J. Differential Equations, 110 (1994), 143-156. 